\title{
Numerical Analysis of Thermally Assisted Magnetization Reversal in Rectangular MRAM Cells Consisted of Exchange Coupled Bilayer
}

\author{
Y. Nozaki, Y. Isowaki, A. Hashimoto, B. Purnama and K. Matsuyama \\ Department of Electronics, Kyushu Univ., Hakozaki 6-10-1, Higashi-ku, Fukuoka 812-8581, Japan
}

Time evolution of magnetization during a cooling process in thermally assisted magnetization reversal of rectangular MRAM cell has been numerically investigated by taking account of not only temperature variations of material parameters but also a thermal fluctuation effect. The rectangular MRAM cell with $100 \times 150 \times 20 \mathrm{~nm}^{3}$ in size is assumed, where the shape anisotropy dominates the switching property. The threshold amplitude of magnetic field required for a successful thermally assisted switching is decreased as the exchange stiffness constant is changed from $1 \times 10^{-6}$ to $1 \times 10^{-7} \mathrm{erg} / \mathrm{cm}$. The time for the magnetization to relax into the bias field direction can be reduced by increasing the bias field amplitude. To switch the magnetization within $1 \mathrm{~ns}$, it is found that the bias field should be larger than 165 Oe. An exchange coupling field in a ferromagnetic bilayer system is considered to be available for producing such a large bias field required for a faster switching in thermally assisted MRAMs.

Key words: thermally assisted, magnetic random access memory (MRAM), numerical simulation, exchange coupling, thin film

\section{Introduction}

Thermally assisted (TA-) magnetization reversal is one of the promising technologies for read/write operations in Gbit-MRAMs, because the competitive demands, i.e. reducing a switching field and ensuring a practical thermal stability, can be simultaneously satisfied by choosing appropriate temperatures in readand write-operations. For the write operation of TA-MRAMs, the magnetization of the cell is switched by cooling in a magnetic field from the magnetic ordering temperature of the storage layer in the MRAM cell. In this study, for the practical application of TA-MRAMs, the temporal evolution of magnetization during the cooling process has been numerically investigated by solving the stochastic Landau-Lifshitz-Gilbert (LLG) equation taking account of not only temperature variations of material parameters but also a thermal fluctuation (TF) effect. For the successful bit-writing, the magnetic ordering due to a bias field application should surmount a thermal disturbance. In this study, the field strength required for successful bit-writing and its minimum switching time have been numerically evaluated for the sub-micron scale MRAM bits. We have also numerically studied about TA-reversal in exchange coupled bilayer system consisting of low- and high- $T_{\mathrm{c}}$ materials. An exchange coupling field in such a system is available for producing a large bias field required for a faster switching in TA-MRAMs.

\section{Model}

The TF effect was approximately included in LLG equations as a randomly directed field with zero mean value. The strength of the random field is evaluated using the fluctuation-dissipation theorem. ${ }^{1)}$ To analyze the stochastic process due to the $\mathrm{TF}$ effect, the each calculation was performed for 50 different series of random field. The Curie temperature, $T_{\mathrm{c}}$, of the cell $\left(100 \times 150 \times 20 \mathrm{~nm}^{3}\right.$ in size) is assumed as $373 \mathrm{~K}$. In this study, an amorphous ferrimagnetic material is considered as a composition of TA-MRAM bits, so that the second-power temperature dependence with the thermally reduced magnetization was assumed both for the exchange stiffness constant, $A$, and the uniaxial crystalline anisotropy, $K_{\mathrm{u}}{ }^{2}{ }^{2}$ The values of the $A, K_{\mathrm{u}}$ and saturation magnetization, $4 \pi M_{\mathrm{s}}$, at $298 \mathrm{~K}$ are $1.0 \times 10^{-7}$ $\mathrm{erg} / \mathrm{cm}, 2.3 \times 10^{4} \mathrm{erg} / \mathrm{cm}^{3}$ and $3.8 \mathrm{kG}$, respectively. ${ }^{3)}$ The temporal variation of the magnetization configuration under the application of bias field, $H_{\mathrm{bias}}$, along easy axis was calculated as the temperature is linearly decreased from 373 to $298 \mathrm{~K}$. The total cooling time is ranged from 0.1 to $50 \mathrm{~ns}$. The Gilbert damping factor is 0.3 , which is typical for the rare earth-transition metal ferrimagnetic alloy. ${ }^{4)}$ The integration time step is fixed as $0.5 \mathrm{ps}$.

To investigate the TA-reversal in exchange coupled bilayer system, it is important to evaluate the temperature variation of the interlayer exchange field, $H_{\text {ex. }}$ In this article, a one dimensional micromagnetic model was adapted to evaluate the $H_{\mathrm{ex}}$ in the bilayer system of $\mathrm{ML}_{2}\left(t_{2}\right) / \mathrm{ML}_{1}\left(t_{1}\right)$, where $\mathrm{ML}_{1}$ and $\mathrm{ML}_{2}$ correspond to low- $T_{\mathrm{c}}$ material such as rare earth-transition metal alloy, e.g. Tb-Fe, and high- $T_{\mathrm{c}}$ one such as transition metal ferromagnet, e.g. Co-Fe, respectively. The saturation magnetization, the uniaxial crystalline anisotropy and the exchange stiffness constant of $\mathrm{ML}_{2}$ are set to be $18 \mathrm{kG}, 3.0 \times 10^{4} \mathrm{erg} / \mathrm{cm}^{3}$ and $1.0 \times 10^{-6} \mathrm{erg} / \mathrm{cm}$, respectively. Corresponding anisotropy field $H_{\mathrm{k}}$ of $\mathrm{ML}_{2}$ is 42 Oe. The material parameters (i.e. $4 \pi M_{\mathrm{s}}, K_{\mathrm{u}}$ and $A$ ) and those temperature dependences for $\mathrm{ML}_{1}$ are same as those mentioned above. ${ }^{3)}$ It is also assumed that the $T_{\mathrm{c}}$ of $\mathrm{ML}_{2}$ is much higher than that of $\mathrm{ML}_{1}$. Therefore, the material 
parameters for $\mathrm{ML}_{2}$ does not change at all when the bilayer is heated up to the $T_{\mathrm{c}}$ of $\mathrm{ML}_{1}$ for the TA-writing. A bilayer system was discretized into one dimensional sub-layer array. The thickness of sub-layer was chosen to be $1 \mathrm{~nm}$, so that the micromagnetic configuration can be interpolated with sufficient number of numerical grids. A continuous magnetization model was adopted for the inter-layer exchange coupling. ${ }^{5}$ ) The second order temperature dependence with the thermally reduced magnetization was adopted for the exchange stiffness constant of the $\mathrm{ML}_{1}$. The field induced in-plane anisotropy was treated as uniaxial. The anisotropy energy and its temperature dependence was estimated from our experimental results for sputtered $\mathrm{Tb}_{23} \mathrm{Fe}_{77} / \mathrm{Cog}_{0} \mathrm{Fe}_{10}$ bilayer films. ${ }^{3}$ ) The perpendicular anisotropy in $\mathrm{TbFe}$ layer is omitted in the present simulation, because the sputtered $\mathrm{Tb}_{23} \mathrm{Fe}_{77} / \mathrm{Cog}_{0} \mathrm{Fe}_{10}$ bi-layer exhibits in-plane magnetization as the thickness of TbFe layer is thinner than $20 \mathrm{~nm}{ }^{3)}$ The switching behavior is, therefore, expected to be dominated by in-plane torque balance especially for a quasi-static case.

\section{Results and discussion}

In the case of $A=1.0 \times 10^{-6} \mathrm{erg} / \mathrm{cm}$, single domain configurations are dominated in the cooled state. The possibility to relax into closure domain configuration is only $2 \%$. The probability relaxing into the positively magnetized state $(50 \%)$ is almost same as that relaxing into the negative one (48 \%), which is reasonable considering a zero mean value of the thermal fluctuation field. Figure 1(a) shows the temporal variation of magnetization component parallel to the easy axis as the temperature decreases from 372.9 to $298 \mathrm{~K}$ for $5 \mathrm{~ns}$. When the $H_{\text {bias }}$ is zero, the magnetization of the particle gradually increases, representing the progress of magnetic ordering. The reduced magnetization is reached to the saturation value at 358 $\mathrm{K}$ (1 ns after cooling). Note that, the magnetization is negatively relaxed in the early stage of cooling $(\sim 0.5 \mathrm{~ns})$ although the positive bias field of 90 Oe is applied. This suggests that the exchange field is dominated to produce the torque exerted on the local magnetization at high temperature near $T_{\mathrm{c}}$. When the reduced magnetization reaches to 0.6 , it seems that the external field gives an effective torque on the magnetization, resulting in the successful TA-switching at $H_{\text {bias }}=90$ Oe [see Fig. 1(a)]. Note that, the threshold amplitude of $H_{\text {bias }}$ required for the TA-switching strongly depends on both the initial magnetization configuration and the random field condition.

In the case of $A=1.0 \times 10^{-7} \mathrm{erg} / \mathrm{cm}$, in contrast, a closure domain configuration is dominated in the relaxation state for zero field cooling. When the reduced magnetization component parallel to the bias field direction, $M_{\text {easy }} / M_{\mathrm{s}}$, reaches to 0.8 , it is found that the

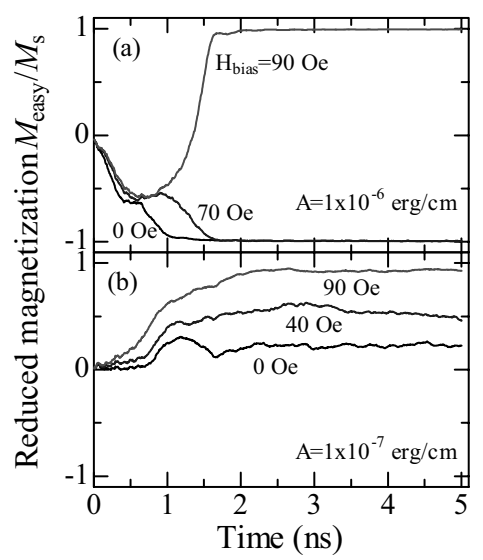

Fig. 1 Temporal variation of reduced magnetization $M_{\text {easy }} / M_{\mathrm{s}}$ during a field cooling process calculated for the particle with (a) $A=1 \times 10^{-6}$ and (b) $1 \times 10^{-7}$ $\mathrm{erg} / \mathrm{cm}$.
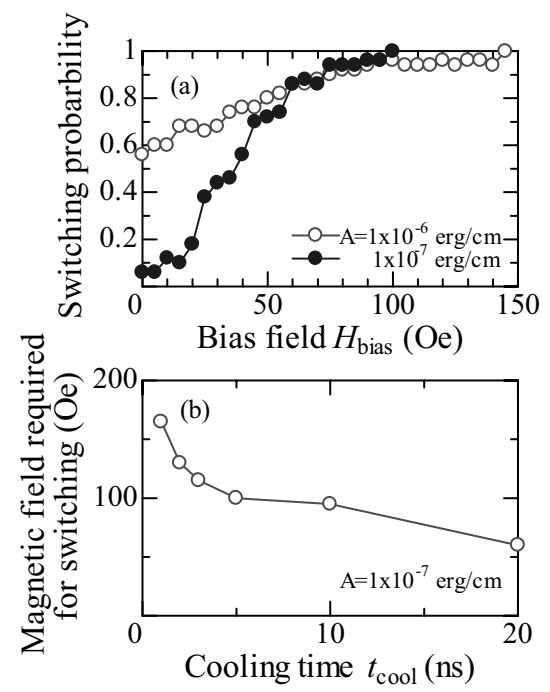

Fig. 2 (a) Switching probability as a function of bias field amplitude and (b) cooling time dependence of the magnetic field required for the thermally assisted switching.

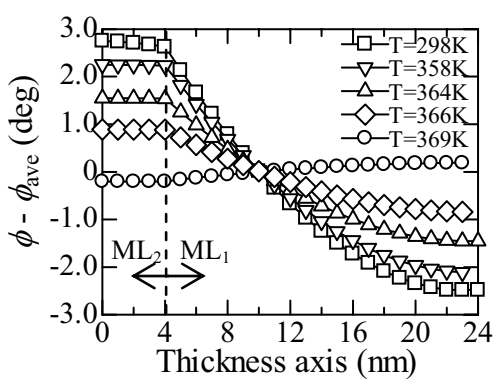

Fig. 3 Variation of the magnetization azimuth angle $\phi$ through the film thickness direction at a timing of the magnetization switching process.

magnetic vortex configuration is almost annihilated from the MRAM cell, so that the magnetization is irreversibly switched along the $H_{\text {bias }}$ direction. The switching probability, $P_{\text {swt }}$, as a function of $H_{\text {bias }}$ calculated for 50 different numerical condition is shown in Fig. 2(a). Here, the occurrence of the switching event 
is judged if the $M_{\text {easy }} / M_{\text {s }}$ exceeds 0.8 or not. The $P_{\text {swt }}$ value is gradually increases with the $H_{\text {bias }}$ and reaches to 1 as $H_{\text {bias }}=100$ Oe for $A=1.0 \times 10^{-7} \mathrm{erg} / \mathrm{cm}$, which is lower than the value for the strongly exchange coupled case $(145 \mathrm{Oe})$. This is attributed to the difference in the magnetization reversal mechanism. In the case of $A=1.0 \times 10^{-7} \mathrm{erg} / \mathrm{cm}, \quad$ a vortex annihilation mainly dominates the switching field, whereas the magnetization is switched via coherent rotation for $A=1.0 \times 10^{-6} \mathrm{erg} / \mathrm{cm}$. Note that, the in-plane demagnetizing factor of the particle plays an important role in both the vortex annihilation field ${ }^{6}$ and the switching field for coherent rotation of magnetization. Therefore, the $H_{\text {bias }}$ required for the TA-switching mainly depends on the shape anisotropy of the particle. Figure 2(b) shows the cooling time dependence of the bias field amplitude required for $P_{\mathrm{swt}}=1$ calculated for the particle with $A=1.0 \times 10^{-7} \mathrm{erg} / \mathrm{cm}$. This result suggests that the larger $H_{\mathrm{bias}}$ is required for the faster bit-writing in TA-MRAMs. To complete the writing process within $1 \mathrm{~ns}$, the $H_{\text {bias }}$ larger than 165 Oe should be applied during the cooling process. For TA-MRAM applications, it is much difficult to get such a large field using the conductor current flowing.

To overcome this difficulty in TA-MRAMs, it is considered that an exchange coupling field in a ferromagnetic bilayer system is available for producing a large bias field required for a faster switching. If the TA-MRAM cell discussed above $\left(T_{\mathrm{c}}=373 \mathrm{~K}\right)$ is exchange-coupled with a soft magnetic layer with the $T_{\mathrm{c}}$ much higher than $373 \mathrm{~K}$, the memory cell can be cooled under the interlayer exchange coupling field, $H_{\text {ex. }}$ For the TA switching in such a system, the exchange coupling field from the magnetic layer consisting of higher $T_{\mathrm{c}}$ material plays an important role for the reordering of the magnetic layer with lower $T_{\text {c }}$. Using a simple one-dimensional model mentioned above, we have numerically evaluated the $H_{\mathrm{ex}}$ in the bilayer system. Figure 3 shows the variation of the magnetization azimuth angle $\phi$ through the film thickness direction at a timing of the magnetization switching process, where the averaged angle, $\phi_{\text {ave, was }}$ 52 degree from the easy axis. Here, the thickness of $\mathrm{ML}_{1}$ and $\mathrm{ML}_{2}$ layers, $t_{1}$ and $t_{2}$, are 20 and $4 \mathrm{~nm}$, respectively. The twisted magnetization configuration is related to the difference of the coercivity in the two layers. The twist angle is about 6 degree at $300 \mathrm{~K}$ and monotonically decreases with the increase of temperature, which can be attributed to the reduced coercivity difference between the two layers. The numerical results reveal that the magnetization switching of the bilayer is occurred simultaneously even at a higher temperature region, despite of the considerable reduction of the exchange stiffness constant. The simultaneous switching behavior enables TA-writing at a temperature lower than the ordering temperature, which would be useful to decrease the power consumption for the cell heating. Rohlsberge et al.

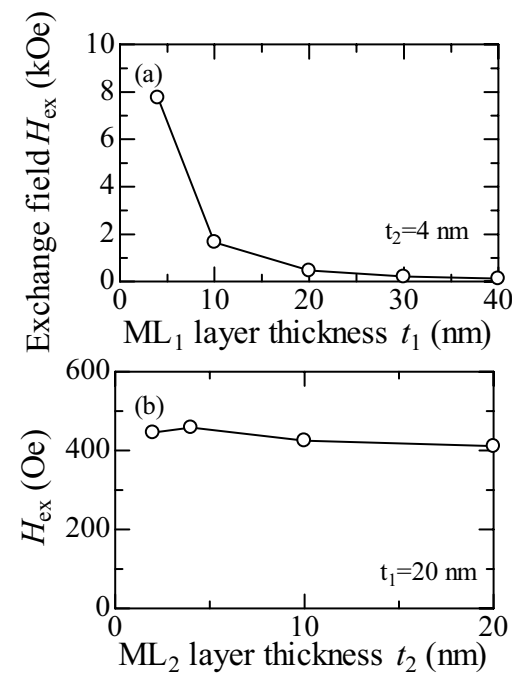

Fig. 4 Interlayer exchange field as a function of (a) $\mathrm{ML}_{1}$ layer thickness and (b) ML2 layer thickness.

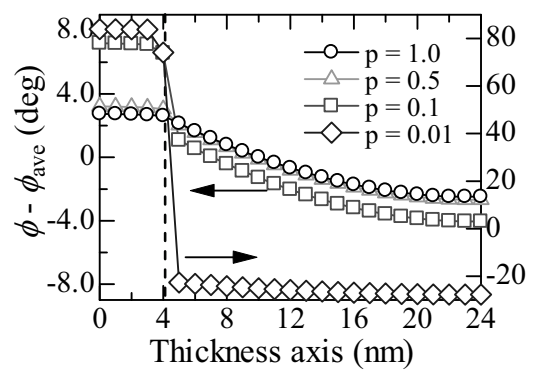

Fig. 5 Variation of the azimuthal angle of magnetization through the thickness direction calculated for the different suppression coefficient of the interlayer exchange coupling.

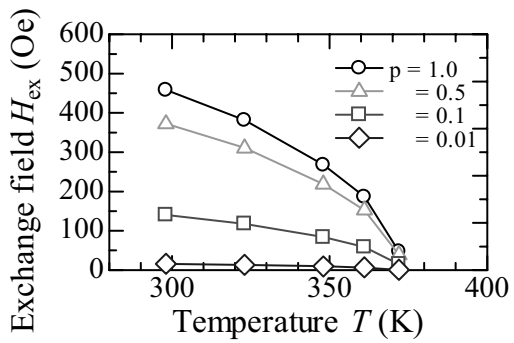

Fig. 6 Temperature variation of exchange field calculated for the different suppression coefficient of the interlayer exchange coupling.

has directly observed such a twisted spin structure in a hard-soft exchange coupled bilayer by nuclear resonant scattering method. ${ }^{7}$ )

As shown in Fig.3, the increase of the exchange energy during the switching process is mainly located inside the $\mathrm{ML}_{1}$ layer consisting of low- $T_{\mathrm{c}}$ material. The $H_{\text {ex }}$ exerted on the $\mathrm{ML}_{1}$ layer can be, therefore, evaluated from $2 E_{\mathrm{ex}} / M_{\mathrm{s} 1}$, where $E_{\mathrm{ex}}$ denotes the density of the exchange energy stored inside the $\mathrm{ML}_{1}$ layer. Figures 4(a) and 4(b) show the evaluated $H_{\mathrm{ex}}$ as a function of $t_{1}$ and $t_{2}$, respectively. The little dependence of $H_{\text {ex }}$ on the $t_{2}$ is attributed to that the increase of $E_{\text {ex }}$ is almost concentrated in the $\mathrm{ML}_{1}$ layer. The amplitude of 


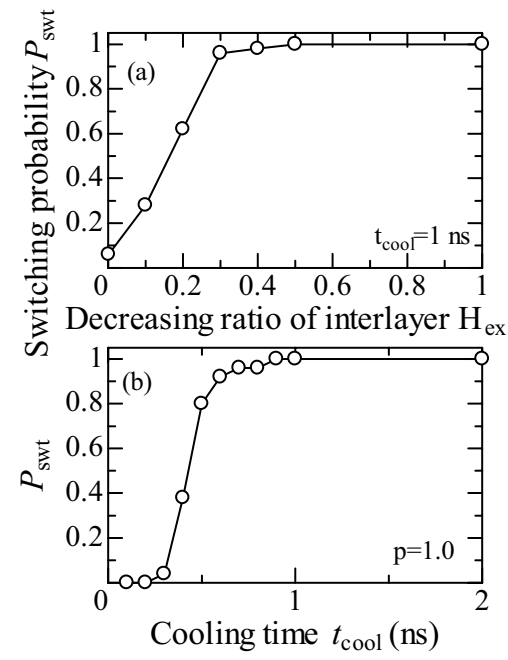

Fig. 7 Switching probability as a function of (a) decreasing ratio of interlayer exchange coupling and (b) the cooling time..

$H_{\text {ex }}$ can be increased with decreasing the $t$. However, in the bilayer system, the $\mathrm{ML}_{1}$ is storage layer so that the decrease of $t_{1}$ causes the suppression of the thermal stability of the bit. The optimization of the $t_{1}$ in the bilayer system is, therefore, most important for the practical TA-MRAM application.

The strength of the interlayer exchange coupling is easily suppressed by the oxidation and/or the atomic diffusion at the interface of the bilayer system. Figure 5 shows the variation of the magnetization azimuth angle through the film thickness direction at a timing of the magnetization reversal calculated for the different suppression coefficient of the interlayer exchange coupling. The temperature is fixed as $298 \mathrm{~K}$. When the suppression coefficient, $p$, becomes smaller than 0.1 , the magnetization of $\mathrm{ML}_{1}$ and $\mathrm{ML}_{2}$ layers are separately switched. Consequently, the amplitude of $H_{\text {ex }}$ at $298 \mathrm{~K}$ is drastically decreased from 458 to 16.2 Oe as the $p$ decreases from 1 to 0.01 .

Figure 6 shows the temperature variation of the $H_{\mathrm{ex}}$ calculated for different $p$ values ranged from 0.01 to 1 . The $t_{1}$ and $t_{2}$ are 20 and $4 \mathrm{~nm}$, respectively. To investigate the TA-switching in bilayer system, the $P_{\text {swt }}$ is calculated for the field cooling process with the application of $H_{\text {ex. }}$. Figure 7 (a) shows the $P_{\text {swt }}$ as a function of suppression coefficient of interlayer exchange coupling. Here, the exchange coupled bilayer consisted of $\mathrm{ML}_{2}(4 \mathrm{~nm}) / \mathrm{ML}_{1}(20 \mathrm{~nm})$ with lateral dimension of $100 \times 150 \mathrm{~nm}^{2}$ is considered. When the $p$ is larger than 0.3 , the magnetization of the particle can be switched along the $H_{\mathrm{ex}}$ direction without applying external field. This result suggests that there may be sufficient robustness in the interfacial characteristics for the application of TA-writing in exchange coupled bilayer system. Figure 7(b) shows the cooling time, $t_{\text {cool }}$, dependence of the $P_{\text {swt }}$ calculated for $p=1$. As the $t_{\text {cool }}$ is decreased below $0.5 \mathrm{~ns}$, the magnetization of the particle tends to relax into a multi-vortices configuration, yielding the write operation error in TA-MRAMs. In the case of $t_{1}=20 \mathrm{~nm}$, it is found that the minimum cooling time required to align the magnetization along $H_{\mathrm{ex}}$ direction is $0.9 \mathrm{~ns}$. In the bilayer system, the magnetization ordering in $\mathrm{ML}_{1}$ is followed by the magnetization switching of $\mathrm{ML}_{2}$. If the $H_{\text {ex }}$ from $\mathrm{ML}_{2}$ is sufficient to set the magnetization of $\mathrm{ML}_{1}$, the TA-switching of bilayer system can be achieved by a low external magnetic field corresponding to the switching field of $\mathrm{ML}_{2}$. To reduce the switching field of $\mathrm{ML}_{2}$, the crystalline anisotropy and the shape anisotropy of $\mathrm{ML}_{2}$ should be as small as possible. As shown in Fig.4(b), the $H_{\text {ex }}$ does not depend on the thickness of $\mathrm{ML}_{2}$. The thickness of $\mathrm{ML}_{2}$ should be, therefore, decrease to minimize its shape anisotropy for the TA-switching of bilayer system with a low operating field.

\section{Summary}

In summary, we have numerically investigated the magnetization reversal of rectangular magnetic particle during the field cooling process. The material parameters of the particle are chosen to be appropriate for the application of thermally assisted switching. For the elongated particle with $100 \times 150 \times 20 \mathrm{~nm}^{3}$ in size, it is found that the bias field larger than 165 Oe should be applied to switch the magnetization within $1 \mathrm{~ns}$. By using the exchange coupled bilayer system as a storage layer of thermally assisted MRAMs, it is confirmed that thermally assisted writing can be successfully performed without applying any external field during the cooling process even if the cooling time is reduced to lower than $1 \mathrm{~ns}$.

Acknowledgements This study was supported by Industrial Technology Research Grant Program in 2005 from New Energy and Industrial Technology Development Organization (NEDO) of Japan.

\section{References}

1) W.F. Brown, Jr.: Phys. Rev., 130, 677 (1963).

2) M. Mansuripur, and G. A. N. Connel, J. Appl. Phys., 55, 3049 (1984).

3) Y. Isowaki, Y. Nozaki and K. Matsuyama, Digest of Intermag'05, EA-11.

4) A. Tsukamoto, K. Nakagawa, A. Itoh, A. Kimel, A. Tsvetkov, H. Awano, N. Ohta, A. Kirilyuk, and T. Rasing, Trans. Magn. Soc. Jpn., 4, 322 (2004).

5) N. Smith, and William C. Cain: J. Appl. Phys., 69, 2471 (1991).

6) K. Yu. Guslienko, V. Novosad, Y. Otani, H. Shima, and K. Fukamichi: Appl. Phys. Lett., 78, 3848 (2001).

7) R. Rohlsberger, H. Thomas, K. Schlage, E. Burkel, O. Luepold, and R. Ruffer: Phys. Rev. Lett., 89, 237201(4) (2002).

Received May 17, 2006; Revised July 14, 2006;

Accepted Aug. 1, 2006 